Saintes corruptions. L'édification romanesque de Jean-Pierre Camus au miroir des adaptations d'Agathonphile (1621)

Barbara Selmeci-Castioni

\title{
CpenEdition
}

Journals

Édition électronique

URL : http://journals.openedition.org/edl/913

DOI : $10.4000 /$ edl. 913

ISSN : 2296-5084

Éditeur

Université de Lausanne

Édition imprimée

Date de publication : 15 décembre 2015

ISBN : 978-2-940331-47-5

ISSN : 0014-2026

Référence électronique

Barbara Selmeci-Castioni, « Saintes corruptions. L'édification romanesque de Jean-Pierre Camus au miroir des adaptations d'Agathonphile (1621) », Études de lettres [En ligne], 3-4 | 2015, mis en ligne le 01 décembre 2018, consulté le 20 décembre 2020. URL : http://journals.openedition.org/edl/913 ; DOI : https://doi.org/10.4000/edl.913 


\section{SAINTES CORRUPTIONS. L'ÉDIFICATION ROMANESQUE DE JEAN-PIERRE CAMUS AU MIROIR DES ADAPTATIONS D'AGATHONPHILE (1621)}

Le roman hagiographique de Jean-Pierre Camus, Agathonphile ou les Martyrs siciliens (1621), décline sur près de huit cents pages le motif de la sainteté et, en contrepoint, celui de la condition déchue. Si les visées édifiantes sont évidentes, la question de la légitimité du recours à la fiction, qui se déploie précisément à la faveur de la corruption qui marque l'homme avant sa conversion, se pose de manière aiguë. Camus prend, comme l'on sait, grand soin à dissiper toute équivoque et, partant, à orienter l'acte de lecture. Il demeure que la séduction d'un lecteur peu disposé à la dévotion est étroitement liée à la représentation de la part sombre de l'humain, dont le roman camusien développe également généreusement les possibles. Les deux adaptations dramatiques de l'Agathonphile, Les Chastes Martirs (1650) de Marthe Cosnard et Agathonphile Martyr (1655) de Françoise Pascal, ainsi que l'abrégé du roman proposé par l'abbé Cusson sous le titre laïcisé d'Agathon et Tryphine. Histoire sicilienne (1712), reconduisent la tension inhérente entre fiction et apologétique; le motif de la corruption permettra d'en mesurer plus spécifiquement les enjeux.

Les choses mêmes les plus saintes ne sont point à couvert de la corruption des hommes; et nous voyons des scélérats qui, tous les jours, abusent de la piété et la font servir méchamment aux crimes les plus grands. Mais on ne laisse pas pour cela de faire les distinctions qu'il est besoin de faire. On n'enveloppe point dans une fausse conséquence la bonté des choses que l'on corrompt, avec la malice des corrupteurs. On sépare toujours le mauvais usage d'avec l'intention de l'art.

Molière, «Préface» de Tartuffe, p. 631. 
Plusieurs décennies avant Molière, Jean-Pierre Camus, évêque de Belley, défend également avec ferveur la nécessité de «sépare[r] toujours le mauvais usage d'avec l'intention de l'art " ${ }^{1}$, l'art étant constamment exposé à la corruption des hommes, soit qu'il en fasse les frais, soit qu'il contribue à en creuser les abîmes. Les intentions du célèbre évêque romancier sont sans conteste édifiantes, mais il est également le premier à admettre que le lecteur est seul maître de l'usage qu'il fait d'un livre, et c'est donc avec d'infinies précautions qu'il mène son entreprise de réformation des mœurs par la rénovation du genre romanesque ${ }^{2}$. Son œuvre, dans la première moitié du siècle, a été couronnée de succès, comme le suggère Perrault qui élève Camus au rang des hommes illustres:

Ses livres passèrent dans les mains de tout le monde, et comme ils étaient pleins non seulement d'incidents fort agréables, mais de bonnes maximes très utiles pour la conduite de la vie, ils firent un fruit très considérable, et furent comme une espèce de contrepoison à la lecture des romans ${ }^{3}$.

Mais que sait-on au juste des différents usages faits de ses livres? Comment appréhender leur effet - édifiant ou corrupteur - auprès du lecteur? Il est une œuvre en particulier qui, à travers ses différentes adaptations, permet de saisir de plus près la réception du projet camusien et, partant, ses nombreux aspects ambivalents: Agathonphile ou les Martyrs siciliens $(1621)^{4}$ a donné lieu à deux versions théâtrales: Les Chastes

I. Voir ci-dessus, la citation mise en exergue.

2. Voir S. Robic-De Baecque, Le salut par l'excès.

3. Ch. Perrault, Les Hommes illustres qui ont paru en France pendant ce siècle, p. 38.

4. J.-P. Camus, Agathonphile, ou les Martyrs siciliens, Agathon, Philargyrippe, Tryphine et leurs Associez. Agathon, Tryphine et Philargyrippe échouent en Sicile après le naufrage de leur vaisseau et échoient, prisonniers chrétiens, entre les mains des païens. L'histoire du roman se déroule pendant leur captivité jusqu'à leur martyre final, sous les persécutions de Dioclétien. Ecrit sur le modèle des romans grecs et baroques, Agathonphile met à profit le temps de l'enfermement pour permettre aux personnages de se raconter mutuellement leurs existences passées par le biais de récits intercalés. Les personnages et le lecteur apprennent ainsi que Philargyrippe, d'un âge vénérable au moment d'effectuer le récit de sa vie, est non seulement un prêtre qui, avant de prononcer ses vœux, était destiné à épouser Deucalie dont il était éperdument amoureux, mais encore, qu'après la mort prématurée de celle-ci, il est devenu objet de convoitise d'une parente de sa fiancée, Nérée, laquelle ne parvenant pas à ses fins finit par calomnier le saint homme et provoquer son exil. Agathon et Tryphine quant à eux sont deux jeunes romains; élevés dans une proximité de voisinage et épris l'un de l'autre, ils prennent 
Martirs (1650) de Marthe Cosnard ${ }^{5}$ et Agathonphile Martyr (1655) de Françoise Pascal ${ }^{6}$, ainsi qu'à un abrégé du roman paru sous le titre d'Agathon et Tryphine, histoire sicilienne, en $1711^{7}$.

Ces adaptations constituent les traces de lectures différentes, d'appropriations personnelles, d'usages pluriels auxquels s'exposait le roman de Jean-Pierre Camus. Or ce roman entretient avec les différents sens de la notion de corruption une périlleuse proximité. Présenté comme "un vaste commentaire estendu en digressions» ${ }^{8}$, Agathonphile, qui s'étend sur près de neuf cents pages, amplifie à souhait quelques lignes du Martyrologe romain et des Annales Ecclésiastiques et peut être envisagé dans une perspective textuelle comme une complexe corruption des sources hagiographiques autorisées ${ }^{9}$. Par ailleurs, en soulignant les débordements passionnels des héros avant leur martyre, le romancier explore également la part corrompue de chacun. Cette double corruption sous-tend également les différentes adaptations du roman. Si leurs auteurs affichent tous une intention édifiante, la sainteté prend à travers ces trois textes des visages différents, sa représentation est altérée jusqu’à devenir méconnaissable. L'exploration de la corruption, à la fois textuelle et morale, est ainsi mise de manière paradoxale au service d'un même projet d'édification dont on peut suivre l'évolution de l'âge baroque à l'orée des Lumières.

la fuite pour échapper à une double menace: les avances de la belle-mère d'Agathon, Irénée, une autre Phèdre, et le mariage forcé qui attend Tryphine avec le vieux patricien Cévère.

5. M. Cosnard, Les Chastes Martirs. Nous utilisons l'édition en ligne établie par H. Fournier, d'après l'édition de 1651: https://uwaterloo.ca/margot/sites/ca.margot/ files/uploads/files/martirs.pdf

6. Fr. Pascal, Agathonphile martyr.

7. J.-B. Cusson, Agathon et Tryphine, histoire sicilienne.

8. J.-P. Camus, «Eloge des histoires dévotes», in Agathonphile, p. 850.

9. Voir J. Le Brun, «La sainteté à l'époque classique et le problème de l'autorisation». 
Altération du texte et corruption des mours: les écueils de la délicate entreprise littéraire de Jean-Pierre Camus

"[...] tout ce qui est au monde, corrompu de malignité, ne vise qu'à la mauvaise Amour ${ }^{10}$ : une telle sentence, sous la plume d'un prélat catholique du premier XVII e siècle n'a rien pour surprendre. Les moyens pour lutter contre la dépravation naturelle de l'homme sont en revanche plus remarquables. Doté d'une conscience aiguë des séductions du littéraire, Jean-Pierre Camus mène, sur le champ des Belles-Lettres, une âpre bataille pour la purification des mœurs à travers une réformation du genre romanesque. Le travail du romancier apparaît cependant sous l'angle d'une altération plurielle, d'une corruption qui se veut salvatrice. Il s'agit, pour reprendre une belle expression de Sylvie Robic-De Baecque, de "divertir d'elle-même la lecture de divertissement" ${ }^{11}$. Parce qu'il est conscient de la difficulté d'une telle entreprise, Jean-Pierre Camus a constamment accompagné ses narrations d'amples préfaces et postfaces destinées à justifier ses stratégies scripturaires et ses projets d'édification fondés sur l'hybridation des matières narratives, religieuses et profanes ${ }^{12}$. Une profonde inquiétude sourd malgré tout à la lecture de ce vaste appareil péritextuel, inquiétude nourrie par la conscience que seule une disposition d'esprit favorable de la part du lecteur peut contribuer à la réussite de son entreprise:

Les Debauchez ne pouvoient appreuver tant de passages de l'Escriture, $\&$ tant de digressions contre le vice, \& en faveur de la Vertu, tournans tout cela en ce suc corrompu dont ils se repaissent. Les Superstitieux d'autre costé treuvent [...] que ce genre d'escrire n'est pas seulement utile mais necessaire pour fournir d'antidote aux Livres deshonnestes, qui corrompent les bonnes mœurs. Mais comme ce sont esprits portez à l'autre extremité, ils ne voudroient pas qu'il y eust tant d'attraicts, ni que l'on approchast si fort l'air des Romans $[\ldots]^{13}$.

IO. J.-P. Camus, «Eloge des histoires devotes», in Agathonphile, p. 892.

II. S. Robic-De Baecque, Le Salut par l'excès, p. 117 et sq.

I2. Voir à ce propos l'anthologie de M. Vernet, Jean-Pierre Camus.

I3. J.-P. Camus, "Dilude» de Pétronille (1628), cité par M. Vernet, Jean-Pierre Camus, p. 140. 
De toutes parts, l'œuvre de Camus s'expose à la corruption, de manière passive ou active. Les parties spirituelles sont menacées par la malveillance des lecteurs les moins dévots, tandis que les parties divertissantes sont susceptibles de servir à rebours le dessein de l'auteur en amplifiant la corruption morale du lecteur. L'inquiétude liée à la réception de son œuvre habite l'auteur dès la parution d'Agathonphile, sa première histoire dévote: "Je n'ay encores redouté la calomnie, [...] comme je fay pour cette Histoire» ${ }^{14}$. Dans l'Eloge des histoires dévotes, il s'empresse de mettre au jour les multiples altérations apportées à ses sources afin qu'on ne puisse les lui reprocher. Le lecteur se trouve ainsi confronté dès le titre à une invention auctoriale, assumée comme telle. Le nom d'“Agathonphile» est en effet revendiqué par l'auteur comme une construction personnelle ${ }^{15}$. Il s'agit d'une hybridation entre les noms des deux héros masculins, Agathon et Philargyrippe ${ }^{16}$. Jean-Pierre Camus n'hésite par ailleurs pas à amender ses sources, qu'elles soient profanes ou religieuses. A propos des poésies mondaines empruntées et modifiées par Camus pour orner Agathonphile, l'auteur admet: "J'auray corrompu la pureté du langage, mais j'auray redressé la pureté des conceptions» ${ }^{17}$. La matière religieuse n'échappe pas aux corrections du romancier:

Que si aux Poesies entierement saines \& sainctes j'ay alteré quelque mot, ce qui est assez rare, c'est pour la raison que j'ay avancee de la varieté des sens que preste la langue saincte, qui est l'originelle des Pseaumes ${ }^{18}$.

Corruption et altération servent ici un même travail d'adaptation stylistique et sémantique des sources, de soumission du texte à l'entreprise littéraire de Camus. La corruption des textes sources profanes, mais également religieux - s'effectue ad majorem Dei gloriam.

I4. J.-P. Camus, «Eloge des Histoires devotes», in Agathonphile, p. 907.

15. Ibid., p. 891 sq.

16. Ce néologisme, pleinement assumé par l'évêque romancier, connaîtra par ailleurs une certaine fortune: en 1649, une Mazarinade tire parti de la notoriété du roman et s'intitule, ironiquement, L'Agatonphile de la France, tandis que l'adaptation théâtrale du roman par Françoise Pascal, sur laquelle nous reviendrons, reprend le composé camusien comme titre, alors même que la pièce renonce au personnage de Philargyrippe.

17. Camus, "Eloge des Histoires devotes", in Agathonphile, p. 889. Nous soulignons.

I8. Ibid., p. 889 sq. Nous soulignons. 
Mais le roman camusien explore également un autre type de corruption, celle qui menace les passions des héros. Pour s'attirer la grâce d'un lecteur amateur de récits chevaleresques et sentimentaux, Camus doit mettre en avant les débordements passionnels de ses héros, fussent-ils appelés à mourir en odeur de sainteté. Que dire de cette main de Tryphine qui suscite la convoitise d'Agathon, et sur laquelle la narration s'arrête avec complaisance? Artifice nécessaire, la peinture des émois suscités par le contact de cette main doit s'entendre in fine comme une allégorie des attraits de «la main de la providence celeste» ${ }^{19}$. S'offusquerait-on de voir Agathon, le futur saint, traverser de nuit une rivière dans le plus simple appareil pour rejoindre sa bien-aimée? L'auteur se contente de rappeler que le héros ne peut s'entretenir avec elle qu' «au travers d'une porte plus espoisse que cent habits ${ }^{20}$. Certes, «les passions ne sont mauvaises qu'autant qu'elles ont un mauvais object, mais elles sont renduës bonnes par un bon" ${ }^{21}$. La frontière est cependant étroite et Camus appréhende qu'on lui reproche d'avoir «escrit des choses amatoires mal à propos» ${ }^{22}$. L'écrivain doit en effet explorer la part corrompue de l'homme, celle qui tend vers la "mauvaise Amour", afin de rendre le triomphe de «l'Amour du bien» encore plus éclatant. Il s'appuie pour cela sur l'exemple des plus illustres péchés: celui de David et Bethsabée, des filles de Loth ou encore de Juda et de Thamar ${ }^{23}$. Mais la peinture de la corruption chez Camus ne possède, évidemment, pas la force de l'autorité biblique: les amours terrestres des futurs martyrs siciliens et de leur entourage relèvent principalement de l'imagination du romancier et n'en sont à ce titre que plus dangereuses, pour le lecteur comme pour l'auteur. Car ces amplifications sont nées de "l'esgayement " ${ }^{24}$ de la plume de l'évêque, qui avoue ainsi à demi-mot qu'il y a en soi un certain

I9. Ibid., p. 855.

20. Ibid., p. 870 .

21. Ibid., p. 860 sq.

22. Ibid., p. 907.

23. Ibid., p. 866. Epouse d'Her, puis de son frère Onan, Thamar doit selon la coutume épouser après la mort de celui-ci le troisième fils de Juda, Séla. Comme Juda diffère cette union de crainte de voir Séla mourir aussi, Thamar se déguise en prostituée et entretient avec son beau-père des rapports charnels sans être d'abord reconnue. Lorsque Juda la reconnait, il s'exclame «Elle a moins de tort que moi». Thamar donne naissance à deux fils, Pharès et Zara (Genèse 38).

24. Ibid., p. 851. 
plaisir à dépeindre la corruption, quand bien même cette peinture ne tend qu'à l'édification du fidèle et à la célébration de l'amour de Dieu.

La sainteté portée "en cette Académie de corruption" ${ }^{25}:$ deux lectures divergentes d'Agathonphile

Malgré les vives critiques dont le théâtre fait l'objet de la part des plus dévots depuis les Pères de l'Eglise ${ }^{26}$, Camus n'eût sans doute pas vu d'un mauvais œil l'adaptation de son œuvre au théâtre, lui qui dès 1632, dans une nouvelle intitulée La Comédienne convertie ${ }^{27}$, appelle de ses vœux une purification de la scène française. S'il s'insurge en particulier contre les farces qui «ne corrompent pas seulement les bonnes mœurs et n'apprennent pas seulement au peuple des mots de gueule, des traits de gausseries et des quolibets sales et déshonnêtes, mais le porte à l'imitation des friponneries et sottises qu'il voit représenter " ${ }^{28}$, il souhaite voir le théâtre, comme le roman, servir à l'édification du peuple: «il serait à désirer que l'ancien usage de la comédie et tragédie, qui était autrefois si célèbre, étant repurgé de tant de défauts et d'impuretés, fût remis en son lustre pour le contentement et l'utilité publique ${ }^{29}$.

Au cœur du XVII siècle, alors que le théâtre connaît dans le sillage de Polyeucte de Corneille une véritable vogue de pièces à martyre, "ce beau Livre intitulé Agatonphile, [où] L'on reconnoist assez l'excellence de son Autheur" ${ }^{30}$ permet à deux dramaturges débutantes de participer chacune à sa manière à la sanctification de la scène profane. Or il est difficile d'imaginer deux lectures, deux usages plus divergents de la même œuvre. Tandis que Marthe Cosnard adapte le roman dévot sur le patron de Polyeucte, surdéterminant par endroit la matière religieuse, Françoise Pascal se concentre au contraire sur les faiblesses et les débordements

25. L'expression est empruntée à François d'Avre qui qualifie ainsi le théâtre dans une "Censure chrestienne du theatre moderne» (Dipne, infante d'Irlande, tragédie, n. p.).

26. A propos des enjeux moraux, poétiques, anthropologiques et métaphysiques de la querelle qui oppose l'Eglise au théâtre au XVII ${ }^{\mathrm{e}}$ siècle, voir L. Thirouin, L'aveuglement salutaire.

27. J.-P. Camus, La Comédienne convertie.

28. Ibid., p. 424.

29. Ibid., p. 425.

30. M. Cosnard, Les Chastes Martirs, "Au Lecteur», [n. p.]. 
passionnels des païens, mais également des futurs saints. Les premières répliques de chacune des pièces emblématisent leurs différences. Le prêtre Philargirippe tient la première place dans Les Chastes Martirs, comme en témoignent la liste des personnages et la première réplique qui lui revient: en dix-huit vers, Philargirippe évoque la "Foy" à trois reprises, ainsi que les figures de saint Paul et de Jésus-Christ, et enfin le sacrement de l'Eucharistie. Dans la pièce de Françoise Pascal, les topoi hagiographiques sont au contraire inversés dès le troisième vers, par le biais de la métaphore amoureuse. Irénée s'exclame ainsi : "ce divin Agathon qui cause mon martyre ${ }^{31}$, et le premier acte est ensuite entièrement consacré à la passion coupable de la marâtre. Les deux épisodes inauguraux - l'arrivée des chrétiens à Syracuse et la scène de séduction d'Irénée - proviennent toutes deux du roman, mais tandis que Marthe Cosnard amplifie en quelque sorte la matière religieuse, Françoise Pascal exalte au contraire le substrat profane.

Les héros des deux pièces, bien qu'il s'agisse des mêmes saints, sont aussi fortement dissemblables. Constant est magnanime dans Les Chastes martirs, Agathon se montre veule et dissimulateur dans la pièce de Françoise Pascal. Aucune des deux dramaturges ne contrevient pourtant fondamentalement à la source camusienne, qui contient une représentation nuancée du futur saint. Marthe Cosnard souligne avant tout les vertus chrétiennes d'Agathon, de Triphyne et de Phylargirippe. Françoise Pascal inverse quant à elle le schéma traditionnel de l'amant conquérant et de la bien-aimée passive, qui informe en partie le roman, au profit d'une représentation qui valorise pleinement l'initiative féminine. Dans le roman, les décisions importantes - telles que la fuite et l'écriture d'une lettre pour tromper les parents de Tryphine - sont le fait du jeune homme. Le dialogue qui préside à la décision de prendre la fuite la veille du mariage forcé de Tryphine possède par exemple la force d'un échange stichomythique:

Mais c'est demain, dit-elle, ce funeste jour, destiné à ma ruine:

$\&$ peut-estre repris-je, sera-ce celuy de votre delivrance.

Ouy, par la mort fit-elle:

Non, Madame, lui dis-je, mais par moy ${ }^{32}$.

3I. F. Pascal, Agathonphile martyr, I, 1, p. 148.

32. J.-P. Camus, La Comédienne convertie, p. 576. Nous modifions la mise en page. 
Le déroulement de la délibération équivalente est tout autre dans la tragicomédie, qui souligne l'inversion des rôles masculins et féminins: c'est Tryphine qui exhorte Agathon à prendre la fuite et, devant la fermeté de la jeune femme, Agathon est forcé d'admettre «Qu'elle fait aujourdhuy l'office d'un Amant" ${ }^{33}$.

Les passions sont plus lisses dans la pièce de Marthe Cosnard. Au cœur de sa démarche esthétique se trouve l'atténuation de la représentation des vices, comme elle l'explique dans l'avertissement au Lecteur:

Je ne me suis arrestée que le moins que j'ay pû dans l'entretien de ces Payens. Et s'il m'eust esté possible de faire des Martyrs sans des Impies, $\&$ voir triompher la Chasteté sans faire parler des infames, Pompone seroit moins criminel, \& son Espouse plus retenuë ${ }^{34}$.

Si elle ne peut faire l'économie de la représentation des opposants, ses personnages sont pour le moins lénifiés en regard de leur source. Par exemple, les débordements affectifs du Préfet et de son épouse sont cantonnés dans les termes de la bienséance; tout au plus y est-il question de «feux» et de "captivité», alors que le roman détaille les nuances de ces différents sentiments. La naissance des passions de Pompone et Elvire est analysée par Camus à travers les fantasmes extraconjugaux qu'elles provoquent auprès des deux époux:

Voyla le Gouverneur \& la Gouvernante blessez en un mesme temps, de mesmes, ou de divers traicts, mesmes puisqu'ils aboutissent à la convoitise, divers à cause des objects. Ignorans la maladie l'un de l'autre, \& esgalement curieux de se celer leur mal, $\&$ desireux de le manifester aux personnes qui le causoient: les voyla en des inquietudes nomparielles, ils souspirent en un même lict pour differentes flammes, $\&$ peut estre qu'ils usent, ou plutost abusent, de ce qui n'est permis qu'au mariage, transportez d'adulteres imaginations $[\ldots]^{35}$.

Si cet héritage est difficile à transposer sur scène, Françoise Pascal ouvre pourtant sa pièce, comme nous l'avons vu, par l'évocation en termes véhéments de la passion illégitime d'Irénée.

33. F. Pascal, Agathonphile martyr, III, 6, p. 198.

34. M. Cosnard, "Au Lecteur», [n. p.].

35. J.-P. Camus, La Comédienne convertie, p. 681. 
Le choix des lieux et l'utilisation de l'espace scénique divergent également entre les deux pièces: tandis que Marthe Cosnard exploite dans les actes III à V le potentiel dramatique et pathétique de la prison, François Pascal structure plusieurs scènes des actes I à IV autour d'un lit, lieu intime des confidences et de la dissimulation. Marthe Cosnard porte une grande attention à la dimension spectaculaire et pathétique de l'espace carcéral, évoqué dans la pièce comme des "Cachots plains d'horreur $\&$ d'effroy " ${ }^{36}$, ce qui est d'autant plus notable que dans le roman les conditions de détention sont plus humaines, presque confortables. L'importance de la prison dans Les Chastes Martirs témoigne du zèle de la jeune dramaturge, sans doute désireuse d'accorder au motif carcéral la même importance que dans Polyeucte, et de susciter par là des effets de terreur et de pitié. On retrouve un zèle similaire chez Françoise Pascal, transposé néanmoins à l'adaptation d'un motif d'une toute autre nature, le lit ${ }^{37}$. Alors que Camus consacre à peine trois pages ${ }^{38}$ à l'épisode de la séduction nocturne d'Irénée, Françoise Pascal lui confère une fonction majeure dans la pièce, puisque le texte suppose la présence scénique d'un lit. Pour rentabiliser en quelque sorte le précieux objet, celui-ci donne également lieu à des scènes pathétiques ultérieures qui ne relèvent plus du roman: la couche d'Agathon devient dans les actes II et IV celle de Tryphine, espace de l'intimité sur laquelle elle se pâme à l'annonce de son mariage forcé avec Cévère, mais également un lieu de dissimulation, qui lui permet de se soustraire à la surveillance de sa suivante pour s'enfuir avec Agathon. Il est intéressant de noter que les deux dramaturges confèrent à ces motifs romanesques secondaires une dimension théâtrale et spectaculaire importante. Leur choix n'est pas anodin et souligne à quel point Marthe Cosnard et Françoise Pascal font un usage différent du roman: la prison sépare le chrétien, porteur de vérité, d'un monde en proie à l'erreur, tandis que le lit figure au cour de la scène l'empire des passions.

36. M. Cosnard, Les Chastes Martirs, IV, 5, v. 764.

37. F. Pascal, Le lict paroist, (I, 2, p. 154); "Il se veut lever, elle l'empesche." (I, 2, p. 155); «Portons-la sur son lict, Pere sans amitié, / Peut-estre cét objet te fera-il pitié.» (Euple, III, 2, p. 183); "Approchons-nous du lict, mais je n'entends personne, / Ha dans quel desespoir le destin m'abandonne.» (Caristée, IV, 2, p. 202).

38. J.-P. Camus, La Comédienne convertie, p. 547-549. 
Le choix des contrées où se déroule l'action est également très significatif. La polarité géographique - entre Rome et la Sicile - était investie par Camus de significations théologiques précises:

Je les appelle Martyrs Siciliens, bien que leur naissance fust Romaine. Et certes le jour de la mort des Martyrs estant appelé Natal par l'Eglise, d'autant que comme celuy qui nous tire des flancs de nos meres pour nous mettre en ce monde, est appellé celuy de nostre nativité; ainsi celuy qui tire l'ame du corps des Saincts par une mort si precieuse, \& si glorieuse que celle que l'on endure pour la Foy, est à bon droit appellé le Natal de l'Eternité ${ }^{39}$.

Le titre alternatif - Agathonphile ou les Martyrs siciliens - souligne la dévaluation de l'origine terrestre des héros (Rome), et met en valeur le lieu de la mort des saints (la Sicile), qui figure leur accession à la sphère divine. La primauté du Natal sur le jour de la naissance corporelle s'inscrit harmonieusement dans le projet édifiant de l'évêque de Belley. Marthe Cosnard reprend le lieu principal du roman: «La Scene est en Siracuse, Ville capitale de Sicile». Françoise Pascal au contraire modifie en profondeur le sujet en déplaçant le lieu du martyre pour le ramener aux alentours de Rome. L'ensemble de la pièce se déroule en effet "à Rome, \& à ses environs». Ce choix désamorce la polarité symbolique entre une Rome terrestre et une Sicile céleste: faire mourir les martyrs près de Rome équivaut sinon à nier, du moins à ne pas prendre en compte la valeur transcendante du lieu de leur sacrifice.

La représentation du martyre dans les deux pièces donne enfin la mesure de l'écart entre une lecture qui privilégie «les enseignements» et l'autre «les faicts " ${ }^{40}$. Préparé dès la première réplique de Philargyrippe, le martyre occupe l'intégralité de l'acte $\mathrm{V}$ dans la pièce de Marthe Cosnard qui suit de près la trame romanesque et conserve des esquisses d'explication théologique (par exemple la réponse de Philargyrippe qui assure Pamphilie que son sang suffira à la baptiser). Au contraire, Françoise Pascal n'accorde que la dernière scène du dernier acte à l'évocation du martyre, greffée in extremis sur une intrigue amoureuse complexe. Après une contestation générale un peu confuse, le père de Tryphine, auquel l'empereur a délégué le pouvoir de faire mourir les chrétiens, invite sans

39. J.-P. Camus, «Eloge des histoires dévotes», in Agathonphile, p. 892.

40. Ibid., p. 924. 
grande conviction les récalcitrants à subir le martyre, et la pièce s'achève sur ces mots de Triphon: "Venez-donc, malheureux, venez souffrir la mort, / Puis que vous le voulez, c'est le dernier ressort ${ }^{41}$. Le martyre se déroule non seulement hors-scène comme dans la majeure partie des pièces hagiographiques de l'époque, mais également hors-texte $-s$ 'il se déroule. Le sacrifice religieux, point d'orgue du roman camusien, ne fonctionne dès lors plus que comme une hypothèse de lecture, une clôture stylistique, qui marque la fin de la pièce hagiographique.

\section{La sainte confondue avec Vénus}

La postérité du roman camusien ne s'arrête pas au milieu du XVII siècle: en 1711, l'abbé Cusson, nouvellement constitué libraire et imprimeur du duc de Lorraine à Nancy, livre un abrégé d'Agathonphile ${ }^{42}$. Ce geste, à la fois auctorial et éditorial, est contemporain des éditions abrégées de l'Astrée, dont Delphine Denis a mis en lumière les enjeux: il s'agit pour les remanieurs de "confirmer la dimension patrimoniale d'une ouvre fondatrice, érigée très tôt en monument» avec un «refus corollaire d'en figer la forme littérale, ce qui serait la condamner à un embaumement certes grandiose, mais qui signerait alors définitivement son avis de décès " ${ }^{43}$. Ce geste engage donc une réflexion sur la place et la forme d'une ouvre littéraire au sein d'une société avec laquelle elle ne partage plus exactement les mêmes codes esthétiques et éthiques. Bien que l'aura du roman camusien soit difficilement comparable à celle de la célèbre pastorale d'Urfé, le projet de Cusson présente des similitudes avec ceux de ses confrères qui s'attachent à rajeunir l'Astrée. Tout en cherchant à prolonger le combat camusien «d'oppos[ition] au cours des Romans \& des autres Livres seducteurs " ${ }^{44}$, il s'agit probablement pour l'abbé-imprimeur d'élever ce célèbre roman dévot à la hauteur d'une œuvre patrimoniale, et de faire en quelque sorte d'Agathon et Tryphine un pendant moralisé de L'Astrée dans le domaine de la littérature d'édification.

4I. F. Pascal, Agathonphile martyr, V, 2, p. 231.

42. Voir supra, note 7.

43. D. Denis, «Bergeries infidèles», p. 27.

44. J.-B. Cusson, Agathon et Tryphine, histoire sicilienne, «Préface», [n. p.]. 
Pour ce faire, l'abbé doit lui aussi altérer le texte original. Afin de "plaire dans ce Siecle, où le goût est si different: on s'est contenté de se servir de la meilleure partie des évenemens qu'il [Camus] y décrit, \& dont on ne peut lui sçavoir trop de gré» ${ }^{45}$. La réduction est conséquente, puisque l'original est ramené à environ un quart de ses proportions initiales. Les épisodes (par exemple celui de la bacchante amoureuse d'Agathon) et les ornements (certains songes, les poésies) probablement jugés superflus au regard de l'action, voire inconvenants, sont retranchés. Si l'architecture générale du roman camusien est conservée, le vocabulaire religieux n'échappe pas à ce travail de modernisation et de simplification stylistique ${ }^{46}$.

La représentation de la sainteté fait également l'objet de modifications qui sont particulièrement frappantes sur le frontispice du livre (fig. 1): les attributs de la sainteté y sont estompés, l'humanité des figures sacrées mise à nu. L'estampe représente le moment suivant le naufrage, celui où les héros échouent sur la rive. On distingue au premier plan deux corps allongés, vêtus à l'antique, au-dessus desquels une jeune femme se tient debout, le regard rivé sur le corps du jeune homme étendu à ses pieds. Autour d'eux, une population agitée. Certains sont munis de bâtons, d'autres agenouillés, les mains jointes. Ce passage illustre un moment extrêmement pathétique, celui où Tryphine croit son bien-aimé Agathon défunt:

Mais quel spectacle pour elle [Tryphine], de le voir ensanglanté, meurtri, déchiré, suffoqué d'eau, \& ne donnant plus aucun signe

45. Ibid.

46. Un exemple ponctuel permet de se faire une idée du minutieux travail stylistique de Cusson, qui devait sans doute procéder, à l'instar de certains remanieurs de l'Astrée, ainsi que l'a montré D. Denis, «ligne à ligne, la plume à la main » ("Bergeries infidèles», p. 23). Le baptême de Deucalie par Phylargirippe, décrit en ces termes par Camus: "comme si elle n'eust attendu que cela, à mesme que je finissois de l'ondoyer, cette ame pure apres ce sacré deluge, comme la blanche colombe du Patriarche, s'envola en l'arche de la celeste patrie, avec le rameau de paix", p. 43, devient chez Cusson: "A mesure que je l'ondoyais, cette Ame pure prenoit son essor; \& la ceremonie achevée, comme la Colombe du Patriarche, elle s'envola au Ciel». Nous soulignons chez Camus les expressions supprimées ou abrégées par Cusson. Un détail mérite par ailleurs d'être souligné: à l'issue du roman, le consul chargé de faire exécuter le décret de Dioclétien, qui se nomme "Calvisian» chez Camus, devient chez Cusson "Cabnisien», estompant par conséquent la référence aux réformés. 


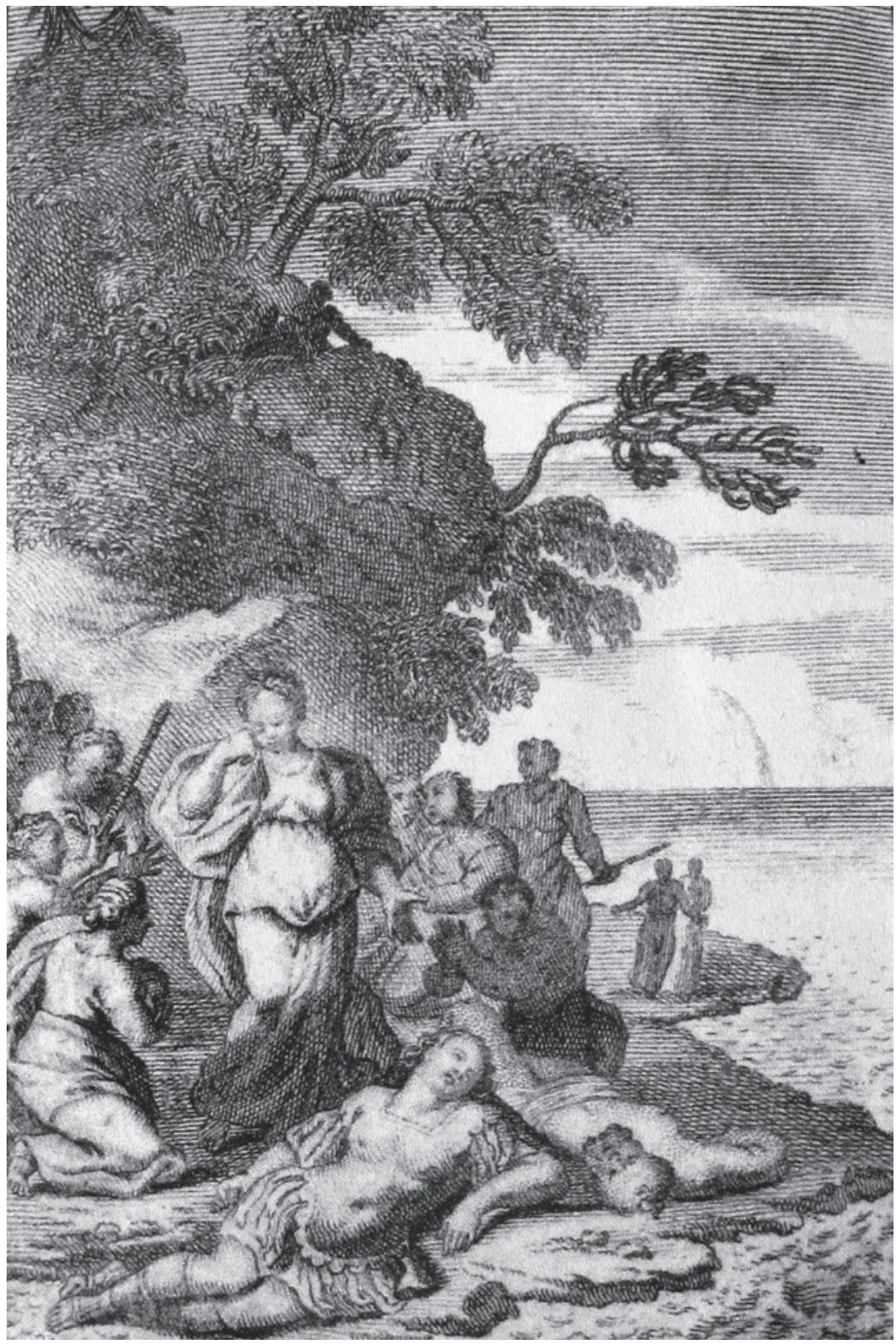

Fig. 1 - Frontispice d'Agathon et Tryphine, histoire sicilienne, de Jean-Baptiste Cusson (1712). 
de vie! Ce fut alors que Tryphine s'abandonna à la douleur, mais à une douleur sage, qui respectoit dans ses malheurs les ordres de la Providence ${ }^{47}$.

Tryphine pleure la perte de l'être aimé, mais plus encore le danger auquel cette perte l'expose:

Quel risque ne court point mon honneur, exposé, dans des lieux inconnus, à la brutalité de ceux qui les habitent? ${ }^{48}$

Ses craintes se voient bientôt confirmées, lorsque les habitants de l'île la confondent avec une divinité:

Ils l'avoient vuë marchant sur les eaux; \& cette circonstance jointe à leur grossiere éducation \& à leur créance, (car la Sicile étoit alors dans les tenebres du Paganisme) leur fit croire que c'étoit Vénus affligée de la perte de quelque nouvel Adonis. Ils se le figuroient d'autant plus aisément, qu'ils sçavoient par tradition, que cette Déesse prit naissance dans le sein de la mer, $\&$ la beauté de Tryphine achevoit de les en persuader $^{49}$.

Lauteur profite de ce passage pour placer dans la bouche de Tryphine des propos dignes du décret tridentin de 1563 sur le bon usage des images saintes:

Bonnes gens, leur dit-elle, que faites-vous? Je n'ai rien de divin [...] ne rendez pas à une vile créature des honneurs qui n’appartiennent qu'à Dieu $^{50}$.

Mais le fait est que la perspective narrative choisie, dans le roman comme dans la gravure, est celle des païens (c'est à travers leur regard stupéfait que le lecteur contemple le naufrage du vaisseau transportant les chrétiens), et cette perspective imite la vision d'un lecteur qui serait aveugle aux marques de la sainteté. A l'exception de la position des personnages qui entourent Tryphine, agenouillés, les mains jointes (encore s'agit-il de païens confondant la sainte avec Vénus!), aucun

47. J.-B. Cusson, Agathon et Tryphine, histoire sicilienne, p. 8.

48. Ibid., p. 9.

49. Ibid., p. 10.

5o. Ibid., p. 11. 
élément de la composition ne permet d'identifier une quelconque dimension hagiographique ou même religieuse. Adopter une telle perspective au début du roman fait certes sens par rapport à la visée apologétique de l'auteur, puisque le roman a précisément pour but de déciller progressivement les yeux du lecteur au fil de l'œuvre. Mais placer l'ensemble du roman sous l'égide de cette perspective profane suggère que, depuis l'évêque de Belley, la distance entre la volonté d'édification et celle de séduction a encore augmenté: la sainteté se camoufle, elle devient méconnaissable. L'adaptation du titre camusien par Cusson - Agathon et Tryphine, histoire sicilienne - efface du reste toute référence martyrologique, nul indice n'informe le lecteur de la sainteté des personnages. Le lecteur est désormais laissé à lui-même pour reconnaître le saint. Quand bien même la corruption ne réside que dans l'œil de celui qui mésinterprète l'apparition - la sainte travestie en Vénus - celle-ci supplante, à l'ouverture du livre, la représentation traditionnelle de la sainteté. L'intention édifiante cède le pas devant la nécessité de séduction.

L'exemple d'Agathonphile et de ses adaptations suggère à quel point l'entreprise littéraire de Camus entretient avec la notion de corruption des rapports de proximité, à la fois nécessaires et gênants, dont l'auteur pouvait appréhender les effets à double titre. D'un point de vue textuel d'abord: Camus admet altérer, voire corrompre ses sources, tant profanes que mondaines. Ces modifications sont d'autant plus périlleuses d'un point de vue orthodoxe qu'elles procèdent de l'imagination, de «l'esgayement» d'une plume épiscopale. D'un point de vue moral, surtout: pour satisfaire l'attente du public visé, le romancier doit explorer minutieusement le cœur de l'homme, même le plus vertueux, qui recèle inexorablement une part de corruption. Les ambivalences, voire les paradoxes du projet camusien apparaissent au grand jour dès lors que l'on compare les différentes adaptations d'Agathonphile. Les deux pièces de théâtre constituent de précieux témoignages des usages religieux et profanes qui pouvaient être faits d'un même texte et montrent que ces deux dimensions ne sont pas imbriquées dans le roman au point d'être inséparables. Tandis que Les Chastes Martirs de Marthe Cosnard constituent la trace d'une lecture dévote, celle sans doute qu'appelait de ses vœux l'évêque romancier, Agathonphile Martyr de Françoise Pascal témoigne d'un usage du livre qui s'attache essentiellement à ses parties romanesques et divertissantes. La version abrégée de Cusson, pour être fidèle en intention à l'entreprise d'édification de l'évêque de 
Belley, ne contribue pas moins à altérer la représentation de la sainteté, en estompant les attributs les plus ostensibles qui rattachaient les figures hagiographiques à l'orthodoxie catholique, ce dont témoigne en particulier le frontispice. De l'âge baroque au début des Lumières, ces textes exploitent des moyens différents pour s'inscrire dans une quête similaire, mettre la sainteté à portée humaine, fût-ce au prix de profondes altérations. Ensemble, ils illustrent les différentes facettes d'un paradoxe littéraire: pour lutter contre «la publication de ces mauvais Escrits, qui ruynent toutes les bonnes mœurs $\&$ fomentent les perverses inclinations de la nature déjà assez corrompuë " ${ }^{51}$, l'auteur aux intentions les plus vertueuses doit également, sur le plan de l'écriture comme sur celui de la moralité, mettre en œuvre différentes formes de corruptions.

Barbara Selmeci-Castioni

Université de Lausanne

5I. J.-P. Camus, «Eloge des Histoires devotes», p. 841. 


\section{BIBLIOGRAPHIE}

\section{Textes}

Avre, François d', Dipne, infante d'Irlande, tragédie, Montargis, Jean Baptiste Bottier, 1668.

Camus, Jean-Pierre, Agathonphile, ou les Martyrs siciliens, Agathon, Philargyrippe, Tryphine et leurs Associez. Histoire dévote où se découvre l'Art de bien Aymer, Paris, Chappelet, 1623 [1621].

- La Comédienne convertie, in Tragédies et récits de martyres en France (fin XVI ${ }^{\mathrm{e}}$-début XVII siècle), éd. par Christian Biet et MarieMadeleine Fragonard, Paris, Classiques Garnier, 2009, p. 423431.

Cosnard, Marthe, Les Chastes Martirs. Tragedie chrestienne, Paris, Courbé, 1650.

Cusson, Jean-Baptiste, Agathon et Tryphine, histoire sicilienne, Nancy, J.-B. Cusson, 1712 [1711].

Molière, Tartuffe, in Euvres complètes, éd. par Robert Jouanny, Paris, Garnier, 1962.

Pascal, Françoise, Agathonphile martyr. Tragi-comédie, éd. par Theresa Varney Kennedy, Tübingen, Gunter Narr, 2008 [1655].

Perrault, Charles, Les Hommes illustres qui ont paru en France pendant ce siècle, éd. par David J. Culpin, Tübingen, Gunter Narr Verlag, 2003.

\section{Travaux}

Denis, Delphine, «Bergeries infidèles: les modernisations de L'Astrée (1678-1733)", Seventeenth-century French Studies, 29 (2007), p. 19-28. 
Le Brun, Jacques, "La sainteté à l'époque classique et le problème de l'autorisation", in Confessionnal Sanctity (c. 1500-c. 1800), ed. by Jürgen Beyer, Mainz, Philipp von Zaberne, 2003, p. 149-162.

Robic-De Baecque, Sylvie, Le salut par l'excès: Jean-Pierre Camus (1584-1652). La poétique d'un évêque romancier, Paris, Honoré Champion, 1999.

Thirouin, Laurent, L'aveuglement salutaire. Le réquisitoire contre le théatre dans la France classique, Paris, Champion classiques, 2007. Vernet, Max, Jean-Pierre Camus: théorie de la contre-littérature, Paris, Nizet, 1995.

Crédit iconographique

Cliché d'après l'exemplaire de la Bibliothèque nationale de France: 8-BL20496. 
